\title{
Antiferromagnetism of almost localized fermions: Evolution from Slater-type to Mott-Hubbard gap
}

\author{
P. Korbel, W. Wójcik \\ Institute of Physics, Technical University, \\ ulica Podchorạżych 1, 30-084 Kraków, Poland \\ A. Klejnberg, J. Spalek \\ Marian Smoluchowski Institute of Physics, \\ Jagiellonian University, ulica Reymonta 4, \\ 30-059 Kraków, Poland \\ M. Acquarone \\ C.N.R.-I.M.E.M., Unita I.N.F.M. di Parma, \\ Dipartimento di Fisica, Universita' di Parma, \\ Parco Area delle Scienze 7A, 43100 Parma, Italy \\ and M. Lavagna \\ Commissariat á l'Energie Atomique, \\ DRFMC /SPSMS, rue des Martyrs, \\ 38054 Grenoble Cedex 9, France
}

October 29, 2018

\begin{abstract}
We supplement (and critically overview) the existing extensive analysis of antiferromagnetic solution for the Hubbard model with a detailed discussion of two specific features, namely (i) the evolution of the magnetic (Slater) gap (here renormalized by the electronic correlations) into the Mott-Hubbard or atomic gap, and (ii) a rather weak renormalization of the effective mass by the correlations in the half-filled-band case, which contrasts with that for the paramagnetic case. The mass remains strongly enhanced in the non-half-filled-band case. We also stress the difference between magnetic and non-magnetic contributions to the gap. These results are discussed within the slave boson approach in the saddle-point approximation, in which there appears a non-linear staggered molecular field due to the electronic correlations that leads to the appearance of the magnetic gap. They reproduce correctly the ground-state energy in the limit of strong correlations. A brief comparison with the solution in the limit of infinite dimensions and the corresponding situation in the doubly-degenerate-band case with one electron per atom is also made.
\end{abstract}


PACS Nos. 71.10.Fd, 75.10.Lp, 75.50.Ee

\section{Introduction}

Antiferromagnetism (AF) appears in the Hubbard and related models for an arbitrary interaction strength $U$ provided we are close to the half-filled-band situation ( $n \rightarrow 1$ in the orbitally nondegenerate case) [1]. This is easy to understand qualitatively, since the intraatomic Hubbard interaction $U \sum_{i}\left\langle n_{i \uparrow} n_{i \downarrow}\right\rangle$ is diminished by keeping apart the electrons with the opposite spins. In the strong-correlation limit 2] this interaction leads to an antiferromagnetic kinetic exchange for an arbitrary band filling $n$. At the same time, the band energy is not increased because the concomitant nesting condition for the quasiparticle states achievable for bipartite lattices only leads to the energy decrease of the occupied states, even when going beyond the Hartree-Fock picture. In effect, the regime of the band filling $n$, for which the AF state is stable at given $U$ has been determined for variety of theoretical approaches [1 - 4]. The reliability of the results for the half-filled case is not in question as long as they reduce to those in the Hartree-Fock and to the mean-field (Heisenberg) approximations in the weak- and strong-correlation limits, respectively, as we will discuss in the following. The theoretical results are in accord with the fact that all known Mott insulators with the half-filled band configuration are also antiferromagnetic insulators. The main purpose of this paper is to overview the situation by discussing the crossover behavior from the Hartree-Fock to the mean-field approximation for the Heisenberg antiferromagnet. Similar, though not equivalent results can be reached within the dynamic mean-field theory (DMFT, see below). In the orbitally degenerate situation (with the degeneracy $d>1$ ) the same type of magnetic(Slater) gap is generated by an alternant orbital ordering [3], as is also discussed at the end of the paper.

Explicitly, we concentrate our attention to two specific features of quasiparticle states not elaborated in detail so far, namely, (i) an evolution of the magnetic gap (renormalized by the electronic correlations) into the Mott-Hubbard gap, and (ii) a rather weak renormalization of the effective mass for the half-filledband case, which is in contrast with that calculated in the paramagnetic (PARA) case 4. A rather strong mass enhancement is retained in the $n \neq 1$ case, in direct analogy to the paramagnetic case [5]. These results are obtained within the slave-boson approach in the saddle-point approximation, which we compare with the corresponding analysis in the infinite-dimension limit 1. In particular, we introduce the concept of a nonlinear staggered molecular field, which shows up as the effective (nonlinear-in-magnetization) magnetic gap, evolving at temperature $T=0$ continuously with increasing $U$ from the Slater (Hartree-Fock) gap $(\sim U)$ into the Hubbard gap. In connection with this evolution, we single out the magnetic and Coulomb parts of the localization energy. These particular features resolve explicitly the old question about the difference between the Slater and Mott-Hubbard insulators in the sense that only the Mott-Hubbard gap survives when antiferromagnetism disappears (i.e. above the Néel tem- 
perature). Most of the results are contained in the mathematical formulation established earlier [1] - 6]. Here, we only discuss those points in an explicit manner. We believe that these points are relevant to the general physics of correlated systems. This reason is also behind publishing, perhaps belatedly, such a simple paper. Additionally, since the effective mass is only weakly renormalized for the $n=1$ case and it never reaches zero for $n \neq 1$, the role of the quantum fluctuations is relatively not as crucial as for the discussion of the paramagnetic state.

The structure of the paper is as follows. In Section II we provide the analytic details of the AF solution, which are followed by the numerical analysis (Section III) and the discussion in physical terms in Section IV, where we also compare the results with those for a doubly degenerate-band case for $n=1$.

\section{Antiferromagnetic solution}

\subsection{Saddle-point approximation}

To stress the role of the molecular field coming from the electronic correlations, we start from the extended Hubbard model, with intersite exchange interactions included, i.e. write

$$
H=\sum_{\substack{i \in A \\ j \in B}} t_{i j} a_{i \sigma}^{\dagger} a_{j \sigma}+U \sum_{i \in A} n_{i_{\uparrow}} n_{i_{\downarrow}}+U \sum_{j \in B} n_{j_{\uparrow}} n_{j_{\downarrow}}+J \sum_{\substack{i \in A \\ j \in B}} \mathbf{S}_{i} \cdot \mathbf{S}_{j}-\mu N_{e} .
$$

where the labels $A$ and $B$ represent two interpenetrable sublattices, each containing half $(N / 2)$ of available atomic sites. The first term represents singleparticle hopping of electrons between the sublattices (nearest neighbors), the second and the third express the intraatomic interaction of the same magnitude on all sites, the fourth includes the Heisenberg exchange between the sublattices, and $\left(-\mu N_{e}\right)$ is the reference energy with $\mu$ being the chemical potential, and $N_{e}(\leq N)$ the total number of fermions. The Heisenberg term has been added only to provide an illustration of the concept of the exchange field coming from the electron correlations (they will add to one another).

In the mean-field (saddle-point) approximation for the slave bosons, the rotationally invariant approach of Li et al. [6] and the original Kotliar-Ruckenstein [1] formulations can be brought to an equivalent form [7. Explicitly, the six bosons $e, p_{\left(\begin{array}{c}A \\ B\end{array}\right) \uparrow}, p_{\left(\begin{array}{c}A \\ B\end{array}\right) \downarrow}$ and $d$ appearing in the approach fulfill the following five constrains in the mean-field approximation

$$
\begin{gathered}
e^{2}+p_{\left(\begin{array}{c}
A \\
B
\end{array}\right) \uparrow}^{2}+p_{\left(\begin{array}{c}
A \\
B
\end{array}\right) \downarrow}^{2}+d^{2}=1, \\
f_{\left(\begin{array}{c}
A \\
B
\end{array}\right) \mathbf{k} \sigma}^{\dagger} f_{\left(\begin{array}{c}
A \\
B
\end{array}\right) \mathbf{k} \sigma}=p_{\left(\begin{array}{c}
A \\
B
\end{array}\right) \sigma}^{2}+d^{2},
\end{gathered}
$$

where the subscript $\left(\begin{array}{l}A \\ B\end{array}\right)$ characterizes the sublattices, and fermion operators $f_{\mathbf{k} \sigma}$ and $f_{\mathbf{k} \sigma}^{\dagger}$ represent the new (quasiparticle) fermion operators appearing in 
the theory. The effective Hamiltonian in the saddle-point approximation takes the form in reciprocal $(\mathbf{k})$ space

$$
\begin{gathered}
H=\sum_{\mathbf{k} \sigma} q \varepsilon_{\mathbf{k}}\left(f_{A \mathbf{k} \sigma}^{\dagger} f_{B \mathbf{k} \sigma}+f_{B \mathbf{k} \sigma}^{\dagger} f_{A \mathbf{k} \sigma}\right)+ \\
\sum_{\mathbf{k} \sigma} f_{A \mathbf{k} \sigma}^{\dagger} f_{A \mathbf{k} \sigma}\left(\lambda_{\sigma}^{(2 A)}-\mu-\frac{1}{2} \sigma J z m_{B}\right)+ \\
\sum_{\mathbf{k} \sigma} f_{B \mathbf{k} \sigma}^{\dagger} f_{B \mathbf{k} \sigma}\left(\lambda_{\sigma}^{(2 B)}-\mu+\frac{1}{2} \sigma J z m_{A}\right)+ \\
N U d^{2}+\frac{N}{2} J z m_{A} m_{B}+H_{c o n},
\end{gathered}
$$

where $H_{\text {con }}$ contains the constraints appearing in the theory in the form of Lagrange multipliers

$$
\begin{gathered}
H_{\text {con }}=\frac{N}{2} \lambda^{(1 A)}\left(e^{2}+d^{2}+p_{A \uparrow}^{2}+p_{A \downarrow}^{2}-1\right)- \\
\frac{N}{2} \lambda_{\uparrow}^{(2 A)}\left[\left(p_{A \uparrow}^{2}+d^{2}\right)+\lambda_{\downarrow}^{(2 A)}\left(p_{A \downarrow}^{2}+d^{2}\right)\right]+ \\
\frac{N}{2} \lambda^{(1 B)}\left(e^{2}+d^{2}+p_{B \uparrow}^{2}+p_{B \downarrow}^{2}-1\right)- \\
\frac{N}{2}\left[\lambda_{\uparrow}^{(2 B)}\left(p_{B \uparrow}^{2}+d^{2}\right)+\lambda_{\downarrow}^{(2 B)}\left(p_{B \downarrow}^{2}+d^{2}\right)\right]
\end{gathered}
$$

The summation over (k) comprises the states in the halved Brillouin zone and $z$ is the number of nearest neighbors. The Lagrange multipliers $\lambda^{(1 A)}$ and $\lambda^{(1 B)}$ correspond to the constraint (2) and, since they are spin symmetric we can put $\lambda^{(1 A)}=\lambda^{(1 B)}=\lambda^{(1)}$. The spin-dependent Lagrange multipliers $\lambda_{\sigma}^{(2 A)}$ and $\lambda_{\sigma}^{(2 B)}$ represent spin-resolved constraint (3). In the antiferromagnetic (AF) case the magnetic moments $m_{B} \equiv\left\langle p_{i B \uparrow}^{2}-p_{i B \downarrow}^{2}\right\rangle$ have the opposite sign, i.e. $m_{A}=$ $-m_{B} \equiv m$. This means that the constraints $\lambda_{\sigma}^{(2)}$ obey the relations $\lambda_{\sigma}^{(2 A)}=$ $\lambda-\sigma^{(2 B)} \equiv \lambda_{\sigma}^{(2)}$. The narrowing factor $q$ renormalizing the bare band energy $\varepsilon_{\mathbf{k}}$ assumes the form

$$
q=\frac{e p_{A \sigma}+d p_{A \bar{\sigma}}}{\sqrt{\left(1-d^{2}-p_{A \sigma}^{2}\right)\left(1-e^{2}-p_{A \bar{\sigma}}^{2}\right)}} \frac{e p_{B \sigma}+d p_{B \bar{\sigma}}}{\sqrt{\left(1-d^{2}-p_{B \sigma}^{2}\right)\left(1-e^{2}-p_{B \bar{\sigma}}^{2}\right)}} .
$$

The Hamiltonian (44) can be diagonalized with the help of the Bogolyubov transformation [1]. In effect, the free energy functional of the system (per atom) takes the form

$$
\begin{aligned}
\frac{F}{N} & =-\frac{k_{B} T}{N} \sum_{\mathbf{k} \sigma} \ln \left[1+e^{-\beta\left(E_{\mathbf{k}}-\mu_{e f f}\right)}\right]-\frac{k_{B} T}{N} \sum_{\mathbf{k} \sigma} \ln \left[1+e^{-\beta\left(-E_{\mathbf{k}}-\mu_{e f f}\right)}\right] \\
& +\frac{1}{2} J z m^{2}+U d^{2}+\mu \frac{N_{e}}{N}+H_{c o n},
\end{aligned}
$$


where now

$$
H_{\text {con }}=\lambda^{(1)}\left(e^{2}+p_{\uparrow}^{2}+p_{\downarrow}^{2}+d^{2}-1\right)-\lambda\left(p_{\uparrow}^{2}+p_{\downarrow}^{2}+2 d^{2}\right)+\Delta m .
$$

Here the quasiparticle energies are $E_{\mathbf{k}}=\sqrt{\left(q \varepsilon_{\mathbf{k}}\right)^{2}+\Delta^{2}}$, with the magnetic gap $2 \Delta=J z m+\left(\lambda_{A \uparrow}^{(2)}-\lambda_{A \downarrow}^{(2)}\right) \equiv \lambda_{\uparrow}^{(2)}-\lambda_{\downarrow}^{(2)}$, and the effective chemical potential $\mu_{\text {eff }}=\mu-\lambda$ with $\lambda=\frac{1}{2}\left(\lambda_{\sigma}^{(2)}-\lambda_{\bar{\sigma}}^{(2)}\right)$. The quantity $\beta_{m}=\frac{1}{2}\left(\lambda_{\uparrow}^{(2)}-\lambda_{\downarrow}^{(2)}\right)=$ $\frac{1}{2} \Delta$ plays a role of the correlation induced molecular field, since it adds to the effective Heisenberg field $J z m / 2$. In the case $J=0$ (taken in the numerical analysis) $\beta_{m}$ constitutes the entire gap induced by the magnetic ordering (it is the magnetic gap). On the whole, the first two terms in (7) provide the contribution to the thermodynamics coming from the single particle excitations in the magnetic (Slater) subbands caused by AF ordering and having energies $\pm E_{\mathbf{k}}$. These quasiparticle energies comprise the effective band narrowing or the mass renormalization $m^{*} / m_{0}=1 / q$, and the molecular field $\beta_{m}$, both to be determined in a self-consistent manner detailed below. The field $\beta_{m}$ arises from the local constraint (31). Thus, one can say that the molecular field is induced by the correlations.

The functional (7) must be minimized with respect to all appearing Bose fields and the chemical potential $\mu$. Effectively, one can reduce (7) to the form with two variables $x \equiv 2 \Delta /(W q)$ and $d$. For the purpose of simplicity and clarity of our points we take constant density of states $(\rho(\varepsilon)=1 / W$ for $-W / 2 \leq \varepsilon \leq$ $W / 2$ ), for which the ground state energy takes the form

$$
\begin{aligned}
\frac{E_{G}}{N} & =-\frac{W}{4} q \sqrt{1+x^{2}}+\left(\frac{1-n}{4}\right) q \sqrt{(1-n)^{2}+x^{2}} \\
& +U d^{2}+\frac{1}{2} m\left(\frac{1}{2} x q-j m\right) W
\end{aligned}
$$

where $E_{G}$ is the ground-state energy, and $j \equiv J z / W$. In this expression we have already connected $m$ to $x$ via the relation

$$
m=x \ln \left|\frac{\sqrt{x^{2}+(1-n)^{2}}-(1-n)}{\sqrt{x^{2}+1}-1}\right| .
$$

For the sake of completness, we list also the explicit form of the band narrowing factor for $n=1$ :

$$
q=\frac{2 \eta}{1-m^{2}}\left[1-2 \eta+\sqrt{(1-2 \eta)^{2}-m^{2}}\right]
$$

Note that the variable $x$ has a physical meaning of the ratio of the Slater gap to the renormalized band energy. In other words, it provides a relative strength of the effective local field with respect to the renormalized kinetic energy. The growing ratio $\Delta /(W q)$ drives the system towards localization induced by the 
formation of staggered magnetic moments arranged in two sublattices, whereas the growing ratio $U / W$ drives the system towards localization independent of magnetic ordering. Therefore, the present formulation will allow us to single out the contributions coming from the two factors. The magnetic energy is thus measured with respect to the band energy $(\sim W q)$ renormalized by the Coulomb interaction.

\subsection{Asymptotic limits: $U \rightarrow \infty$ and $U \rightarrow 0$}

We now show that the results obtained above provide correctly a mean-field solution in the $U \rightarrow \infty$ limit. For the sake of simplicity consider the halffilled band case with $J=0$ and for symmetric form of the density of states, $\rho(\epsilon)=\rho(-\epsilon)$. We can then write the ground state energy (per site) in the form

$$
\frac{E_{G}}{N}=-2 \int_{-W / 2}^{0} d \epsilon \rho(\epsilon) \sqrt{(q \epsilon)^{2}+\Delta^{2}}+U d^{2}+\Delta m .
$$

For large $U$, the gap $\Delta$ is also large. In that limit the energy has the form

$$
\frac{E_{G}}{N}=-\frac{q^{2}}{\Delta}<\epsilon^{2}>+U d^{2}+\Delta(m-1),
$$

where $<\epsilon^{2}>\equiv \int_{-W / 2}^{0} d \epsilon \rho(\epsilon) \epsilon^{2}$. Minimizing this expression with respect to $\Delta$ we obtain that

$$
m \simeq 1-\left(\frac{q}{\Delta}\right)^{2}<\epsilon^{2}>.
$$

Minimization with respect to $m$ and $d^{2}$ gives the relations

$$
\frac{2 q_{m} q}{\Delta^{2}}<\epsilon^{2}>=1
$$

and

$$
\frac{2 q_{d^{2}} q}{U \Delta}<\epsilon^{2}>=1
$$

where $q_{x}=d q / d x$. Combining Eqs.(14)-(16) we obtain that $q=2(1-m) q_{m}$, and $\Delta=\left(q_{m} / q_{d^{2}}\right) U$. Explicitly, from the fact that $q \simeq 2 \eta /(1-m)$ and that numerically $q \approx 1$, we obtain that $d^{2} \simeq(1-m) / 2$, and $\Delta \approx U / 2$. Thus finally, for the featureless form of the density of states we have

$$
\begin{aligned}
& m=1-4 \frac{<\epsilon^{2}>}{U^{2}}=\frac{1}{6}\left(\frac{W}{U}\right)^{2}, \\
& d^{2}=2 \frac{<\epsilon^{2}>}{U^{2}}=\frac{1}{2}\left(\frac{W}{U}\right)^{2},
\end{aligned}
$$

and

$$
\frac{E_{G}}{N}=-2 \frac{<\epsilon^{2}>}{U}
$$


In other words, in the $U \rightarrow \infty$ limit the Hubbard model reduces to the Heisenberg model [2] with the Hubbard gap $\Delta=U$, since in the mean-field approximation the ground-state energy is then given by the kinetic exchange contribution 2

$$
\begin{aligned}
\frac{E_{G}}{N} & =-\frac{4}{U N} \sum_{i_{A} j_{B}} t_{i_{A} j_{B}} t_{j_{B} i_{A}}\left\langle\mathbf{S}_{i} \cdot \mathbf{S}_{j}-\frac{1}{4}\right\rangle \\
& \approx-\frac{2}{U N} \sum_{i_{A} j_{B} \sigma} t_{i_{A} j_{B}} t_{j_{B} i_{A}}=-\frac{1}{U(N / 2)} \sum_{\mathbf{k}} \epsilon_{\mathbf{k}}^{2} \\
& =-\frac{1}{U} \int_{-W / 2}^{0} d \epsilon \epsilon^{2} \rho(\epsilon) \equiv-2 \frac{\left\langle\epsilon^{2}\right\rangle}{U}
\end{aligned}
$$

with $\rho(\epsilon)=\frac{1}{N / 2} \sum_{\mathbf{k}} \delta\left(\epsilon-\epsilon_{\mathbf{k}}\right)$. Also, the magnetic gap reduces to the atomic value of the Hubbard gap, as $2 \Delta \rightarrow U$ with $\left\langle\epsilon^{2}>/ U \rightarrow 0\right.$.

The Hartree-Fock $(U \rightarrow 0)$ limit is recovered once one notices that the method has been constructed to obtain $q=1$ in the weak coupling limit [6], [7]. Under that circumstance Eq. (9) reduce to the usual Hartree-Fock form if we assume that now $x=2 \Delta / W \ll 1$. This limit was checked out also numerically, but the results are not reproduced here.

\section{$3 \quad$ Numerical analysis}

In Fig.1 we have displayed both the effective magnetic (Slater-type) gap $2 \Delta$ and the Mott-Hubbard gap for the paramagnetic phase, both for $n=1$ Those characteristics are plotted for the ground state. The chemical potential is then $\mu(T=0)=\lambda$. The Mott- Hubbard gap is expressed through the difference in the chemical potential in the paramagnetic case $(\Delta=0)$ for $n=1.001$ (the upper part) and for $n=0.999$ (the lower part) and was discussed earlier [8]. For $n=1$ the Slater split-band picture appears for arbitrary small $U$ and $\Delta$ increases with increasing $U / U_{c}$, where $U_{c}=8|\bar{\epsilon}|$. In the limit $U / U_{c} \sim 1$ the gap is composed of the Slater and the Mott-Hubbard parts, and when $\rightarrow \infty$ the former merges gradually with the latter. This can be seen explicitly in Fig.2, where we have shown the ground state energy $E /(W N)$ versus $U / U_{c}$. In the strong-correlation limit the energy is determined by the kinetic-exchange contribution $\sim 1 / U$ [2] as shown explicitly. The fitted coefficient $\alpha=0.042$ to the numerical results cannot be attributed to any particular $3 \mathrm{~d}$ structure, since we have used in numerical calculation a constant value of $\rho(\epsilon)$. The energies of para- and antiferro-magnetic states merge for $n=1$ and in the $U \rightarrow \infty$ limit. The inset illustrates another interesting characteristic of the solution, namely the magnetic gap is not proportional to the magnetization, as one would expect from the Hartree-Fock (Slater) solution. In other words, the molecular field $\beta_{m}$ is a nonlinear function of $m$, since from the condition $\partial f / \partial \Delta=0$ we obtain the

relation $2 \Delta / W=m q / \sqrt{1-m^{2}}$. This is the reason why the antiferromagnetic 


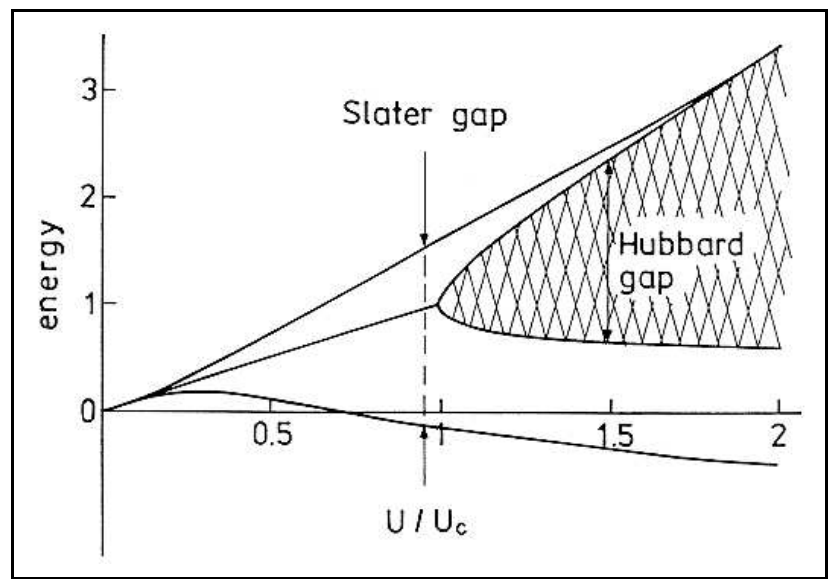

Figure 1: The Slater gap $2 \Delta / W$ and the Hubbard gap as the functions of interaction strength. The Slater gap merges with the Mott-Hubbard gap as $U / U_{c} \rightarrow \infty$.

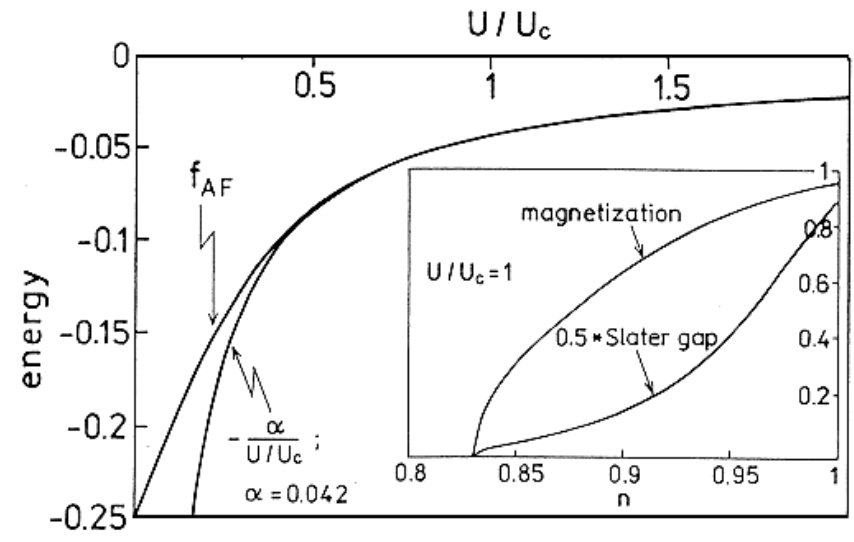

Figure 2: Ground state energy for $\mathrm{AF}$ state and $n=1$ versus $U / U_{c}$. The lower curve is the fit to the expression $\left(-0.042 \times U_{c} / U\right)$. The inset displays the difference in behavior of magnetic moment $m$ and half of the Slater gap $(\Delta / W)$, both plotted as a function of the band filling. 


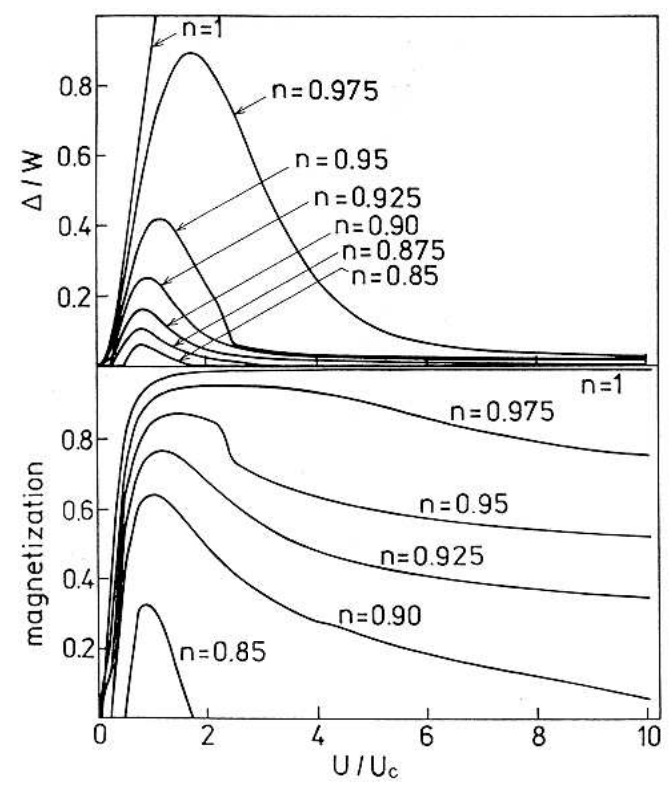

Figure 3: The Slater gap parameter $\Delta / W$ (top panel) and the magnetic moment $m=\left\langle n_{i \uparrow}-n_{i \downarrow}\right\rangle$ (bottom) versus $U / U_{c}$ and for different values of $n$.

gap for almost localized fermions cannot be regarded as just the Slater gap. Also, the AF solution disappears at the critical band filling $n \equiv n_{c} \simeq 0.83$.

To visualize the difference between the magnetic gap and the magnetization we have plotted in Fig. 3 both quantities as a function of the interaction strength $U / U_{c} \equiv U / 2 W$ for different band filling parameter $n$. While for $n=1$ the magnetic moment saturates gradually with growing $U / U_{c}, \Delta$ keeps increasing. The magnetic gap for $n=1$ increases and eventually $\Delta \sim U$; this circumstance indicates again that the magnetic gap merges with the Hubbard gap, which can be estimated analytically and is $\approx-W+4 U W^{2} /\left(z\left(U^{2}+W^{2}\right)\right) \rightarrow U(1-W / U)$ for $W / U \rightarrow 0$.

The double occupancy probability $d^{2}=\left\langle n_{i \uparrow} n_{i \downarrow}\right\rangle$ is shown in Fig.4 for different band fillings. It decreases continuously with growing $U / U_{c}$, i.e. the charge fluctuations are gradually suppressed, while the magnetic moment grows (cf. Fig.3). The difference in the behavior of $d^{2}$ and $m$ is caused by the circumstance that the $d^{2}$ is of intraatomic nature, whereas $m$ is determined from the competition between the magnetic energy $\sim \beta m^{2}$ (also of intraatomic nature) and the renormalized band energy $\sim W q$. The inset to Fig.4 exemplifies the difference between the diminution of $d^{2}$ with growing $U / U_{c}$ for $n=1$ in two situations: For the paramagnetic (PARA) case $d^{2} \equiv 0$ for $U \geq U_{c}$; this feature is concurrent with the well-known effective mass divergence at the Mott-Hubbard localization boundary (the Brinkman-Rice point). This divergence does not emerge in the 


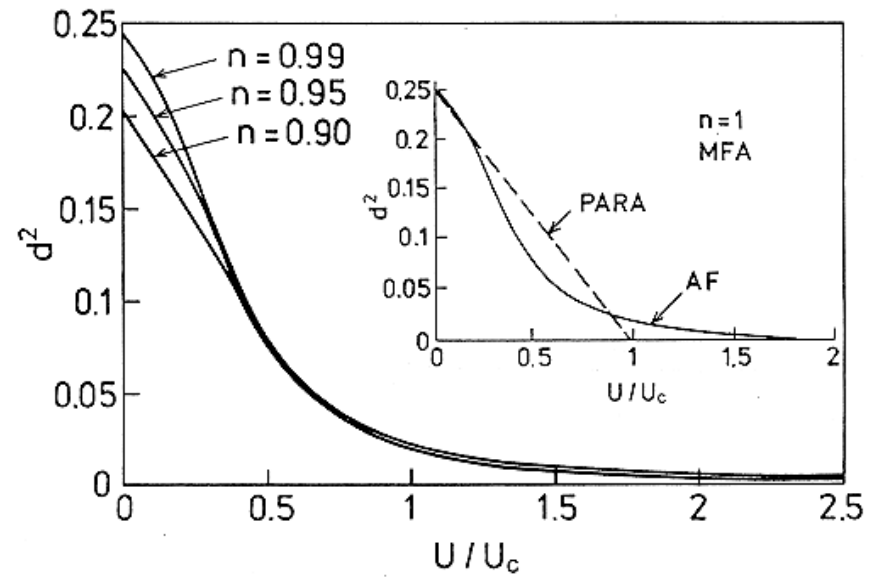

Figure 4: The double occupancy $d^{2}=\left\langle n_{i \uparrow} n_{i \downarrow}\right\rangle$ vs $U / U_{c}$, and for the different $n$ values. The inset display the difference in behavior for para- and antiferromagnetic cases for $n=1$.

antiferromagnetic state as $d^{2}$ approaches zero gradually, in the same manner, as $m$ approaches saturation $m \rightarrow 1$ ).

In Fig.5 we have displayed the inverse band narrowing factor $q^{-1}$ (for $n=1$ ) which turns into the effective mass renormalization $m^{*} / m_{0}=1 / q($ for $n<1)$ as a function of $/ U_{c}$ and for different $n$ values. Again, the inset illustrates the difference with the $n=1$ case. One should note that the enhancement factor in AF state is very small compared to that in PARA state, which is equal to $1 / q=\left[1-\left(U / U_{c}\right)^{2}\right]^{-1}$. The difference between AF and PARA states diminishes with decreasing $n$, as in that situation the magnetic moment is reduced rapidly. So, the weak band narrowing in the $n=1$ case can be associated with the circumstance that the Fermi level falls in the gap. This is the reason why $m^{*} / m_{0}$ raises rather steeply around $n \simeq 0.95$. Thus, the appearance of the itinerant antiferromagnetism with an isotropic gap changes the Brinkman-Rice scenario for the Mott-Hubbard transition, as it has been underlined before [9]. Also, the physical parameters $d^{2}, 1-m$ and $1-n$ are all of the same magnitude. This is easy to envisage when estimating e.g. the band narrowing $q$, which is in the AF state roughly $\operatorname{sim} 2 d^{2} /\left(1-m^{2}\right)$ and is of the order of unity.

In Fig. 6 we have displayed the stability regime $\left(n, U / U_{c}\right)$ of the AF phase. The full circles has been obtained [10] in the limit of infinite dimension with the help of quantum Monte Carlo simulation. One should note the range of the filling $n$ of stable AF phase is the broadest for $U / U_{c} \sim 1$, i.e. when the molecular field is the strongest (cf. Fig.3). Note also that the Monte-Carlo results did note provide the asymptotic behavior for $n \rightarrow 1$, as it does not reduce correctly to the Hartree-Fock limit. 


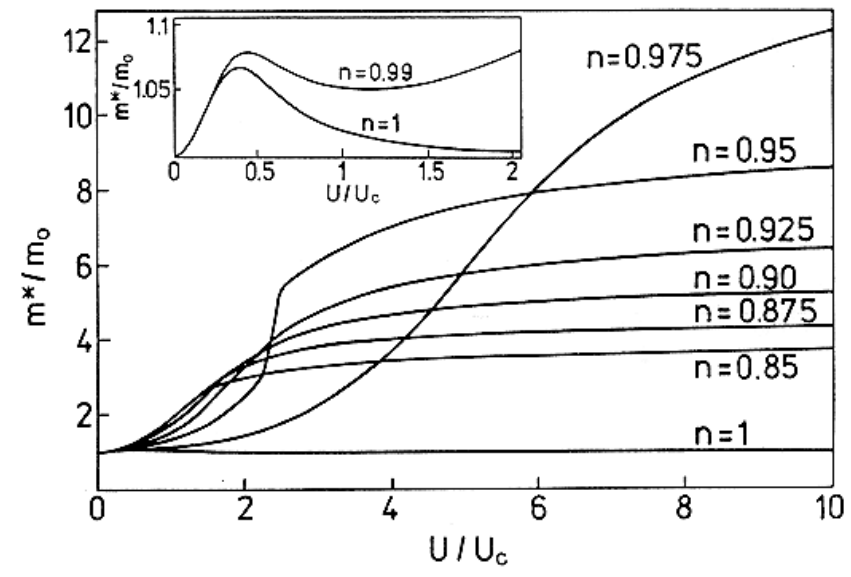

Figure 5: The effective mass enhancement $m^{*} / m_{0}$ (with respectto the band value $\left.m_{0}\right)$ vs $U / U_{c}$ and for the $n$ values shown. The inset shows a rather weak enhancement close to the Mott-Hubbard limit.

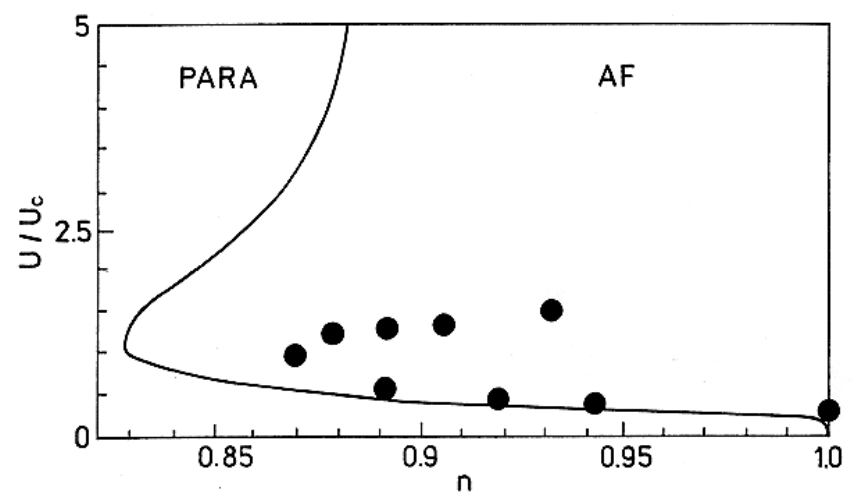

Figure 6: The stability regime of AF solution; the inset: results of Monte-Carlo calculations in the $d \rightarrow$ limit [8] 


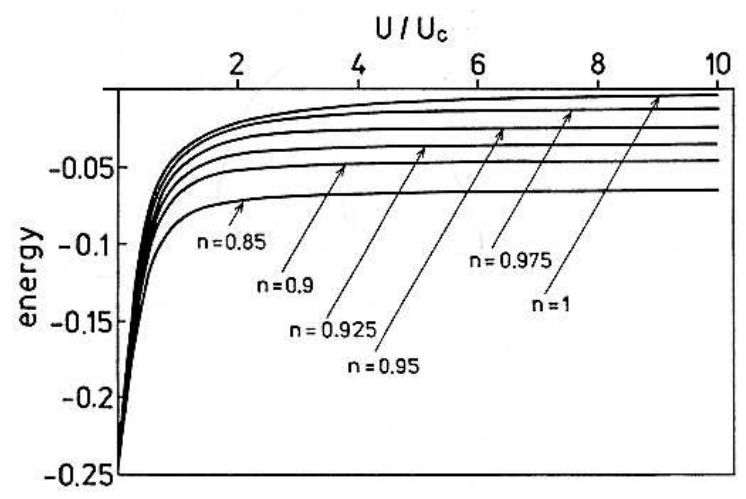

Figure 7: Ground state energy vs $U / U_{c}$ for different band filling $n$. In each case the energy is $\sim U$ for $U \lesssim 0.5 U_{c}$, and $\sim 1 / U$ for $U>U_{c}$.

For the sake of completeness we display in Fig.7 the ground state energy as a function of $U / U_{c}$, for different $n$ close to the half filling. In each case (for $n<1)$ the system contains the contribution $\sim U$ for $/ U_{c} \lesssim 0.5$ and the contribution $\sim 1 / U$ for $U / U_{c}>1$. In other words, the solution interpolates between the Hartree-Fock and the kinetic exchange limits. Moreover, the shift of the curves with diminishing $n$ in the $U \rightarrow \infty$ limit is due to the presence of the band term proportional to $n(1-n)$. One should note that in the paramagnetic state the direct Coulomb interaction contribution $\left(U d^{2}\right)$ competes with band energy $(-W q / 4)$. In the antiferromagnetic state the local magnetic contribution $\left(-\left|\beta_{m}\right| m\right)$ is of the opposite sign than the Coulomb part. However, it changes also the band energy. To specify the role of the staggered field we have plotted in Fig.8 the difference $\left(U d^{2}-\left|\beta_{m}\right| m\right) / W$ as a function of $U / U_{c}$. The magnetic contribution exceeds the Coulomb part for $U>0.5 U_{c}$ (i.e. away from the Hartree-Fock limit), and is particularly strong (and comparable to the band energy) in the regime $U \sim U_{c}$, where the Mott-Hubbard localization would take place for the paramagnetic case. This circumstance indicates why the metal-insulator transition present at $T=0$ in the paramagnetic phase is wiped out when the itinerant AF sets in. Simply, the magnetic contribution $\left(\sim \beta_{m} m\right)$ freezes gradually the spatially separated electrons in the antiferromagnetic phase, taking also advantage of nonvanishing kinetic energy (the kinetic exchange, $\sim<\epsilon^{2} / U$, contribution!). In the PARA phase we have instead a direct competition between band and Coulomb energies.

To summarize, the Mott-localization is achieved gradually at $T=0$ in the AF state. In other words, the present approach provides a crossover behavior from Slater to Mott insulator, as shown e.g. in Fig.2. The same holds true even when we include the intersite exchange $(J>0)$, which may originate from the quantum Gaussian fluctuations. The continuous evolution with growing $U / W$ does not preclude the first order transition at nonzero temperature, as has 


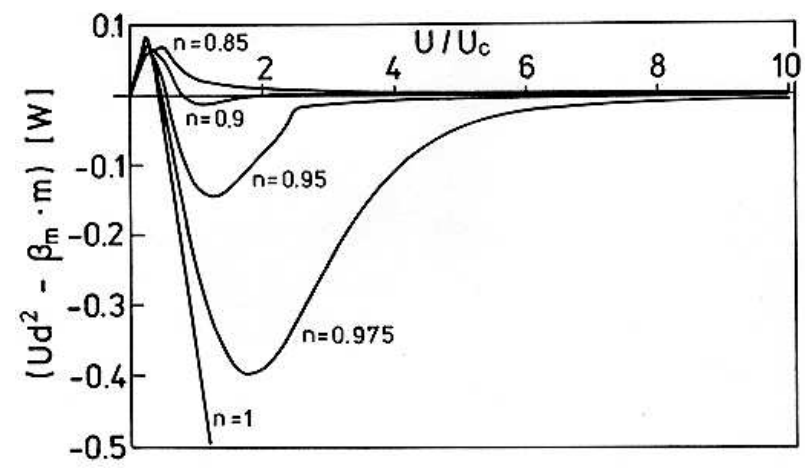

Figure 8: Comparison of the magnetic $\left(-\beta_{m} m\right)$ and the Coulomb $\left(U d^{2}\right)$ terms in the ground state energy versus $U / U_{c}$.

been demonstrated some time ago for the paramagnetic state 11 and subsequently reconfirmed in the limit $d \rightarrow \infty$ 12. The correlated state builts in gradually with increasing $U / U_{c}$, as can be seen from the gradual increase of the mass enhancement in $n<1$ case, i.e. when the ground state is always metallic. The same holds true for the ground state energy shown in Fig.7.

\section{Discussion and conclusions}

We have addressed the question of crossover from Slater (Hartree-Fock) to MottHubbard (atomic) picture in the half-filled band case, as well as have analyzed the behavior of quasiparticle physical properties in AF state in the half- and non half-filled-band cases. Although our analysis is based on the saddle-point solution within the slave-boson functional-integral approach, the results can serve as a mean-field analysis, since they interpolate between those in the Hartree-Fock approximation in the limit $U \rightarrow 0$ and those in mean-field approximation for the Heisenberg model (for $n=1$ ) in the $U \rightarrow \infty$ limit. They also represent basis for inclusion of Gaussian fluctuations [13] in a magnetically ordered (AF) state close to the Mott-Hubbard localization. However, one should realize that for the half-filled band case the effective mass is only weakly renormalized so the renormalization factor $z_{k_{F}}=\left(m_{0} / m^{*}\right) \sim 1$. Also, for $n \neq 1, z_{k_{F}}$ remains always finite so, perhaps the role of the quantum fluctuations is not as crucial for $\mathrm{AF}$ state, as it is for the paramagnetic state. In any case, it will be much more involved that in the paramagnetic case 13 . The full analysis of the MottHubbard boundary should also include the disordered local-moment phase [14, so far not included within the present scheme.

The physical meaning of the results obtained within the slave-boson approach (SBA) can be characterized as follows. It is well known [1], [6] - 8], [13] that the saddle point solution of the approach reproduces the results of 
the Gutzwiller approach (GA), as far as the overall (ground-state) behavior is concerned. Moreover, SBA leads to an improvement of the GA by incorporating both the quasiparticle picture of those systems and the quantum Gaussian fluctuations (not considered in this paper). In general, the pseudo-fermion fields $\left\{f_{i \sigma}\right\}$ representing the quasiparticle states are in one-to-one correspondence to the original fermion fields in the physical Fock space. The mapping is quite obvious within the above analysis. Specifically, the quasiparticle energy $E_{\mathbf{k}}=\sqrt{\left(q \epsilon_{\mathbf{k}}\right)^{2}+\Delta^{2}}$ leads to the following density of states

$$
\rho(E)=\left.\frac{2|E|}{\sqrt{E^{2}-\Delta^{2}}} \rho^{0}(\epsilon)\right|_{\epsilon=\sqrt{E^{2}-\Delta^{2}}},
$$

where $\rho^{(0)}(\epsilon)$ is the bare density of states in PM phase. Thus the enhancement factor due to the correlations is distinct (and disappear in the first factor) from the change of the density of states caused by the appearance of the magnetic gap. By analogy, for the paramagnetic state $(\Delta \rightarrow 0)$, for which $E_{\mathbf{k}}$ can be written as

$$
E_{\mathbf{k}}=q \epsilon_{\mathbf{k}} \equiv \epsilon_{\mathbf{k}}+\Sigma\left(\epsilon_{\mathbf{k}}\right),
$$

and where the self-energy $\Sigma(\omega)=(1-q) \omega$ leads to the mass enhancement $m^{*} / m=(1-\partial \Sigma / \partial \omega)^{-1}=1 / q$, we cannot single out the factor $(1 / q)$ in (21) as the corresponding enhancement also in AF state. In the doubly-degenerate-band case (for $n=1$ ) and under the same-type of approximation scheme, the role of the magnetic gap is played by the gap formed by an alternant orbital ordering in the ferromagnetic state [3]. The gap $(\delta)$ in the latter case is reproduced in Fig.9 for the magnitude of the intraatomic (Hund's-rule) exchange $J / U=0.2$. Again, the gap approaches asymptotically the Mott-Hubbard gap, which in this case [3] is equal to $(U-3 J) / 2$. However, here there is a critical value of $U / W$, at which the system exhibits antiferromagnetic orbital ordering. In the half-filled case for the doubly degenerate case $(n=2)$ the gap appears for an arbitrary small $U$ [15] in the AF case and for the critical value of $U$ for the paramagnetic phase [15.

One should also note that within the present SB scheme the antiferromagnetic Slate-type state (AFS) evolves for $n=1$ gradually into antiferromagnetic Mott (AFI) state with increasing $U / W$ ratio. For $n<1$, we observe only antiferromagnetic metallic (AFM) state. This is in contrast with the results obtained with the composite-operator method [16, as well as with an original analysis [17] where a phase border line between itinerant- and localized-staggered momentbearing phases is drawn. Localized-moment phase for $n=1-\delta, \delta<<1$ can be obtained only when the polaronic effects due to antiferromagnetic moments surrounding the hole in the Mott insulator are included [18. Obviously, such ASF-AFI border line at temperature $T>0($ for $n=1)$ is present [11] and is induced by the difference in entropy of AFS and AFI states.

The computational details of the present work are available on request [19].

Acknowledgment J.S. is grateful to Claudine Lacroix and M. Avignon for discussions during his stay at DRFMC-CENG in Grenoble. This research was 


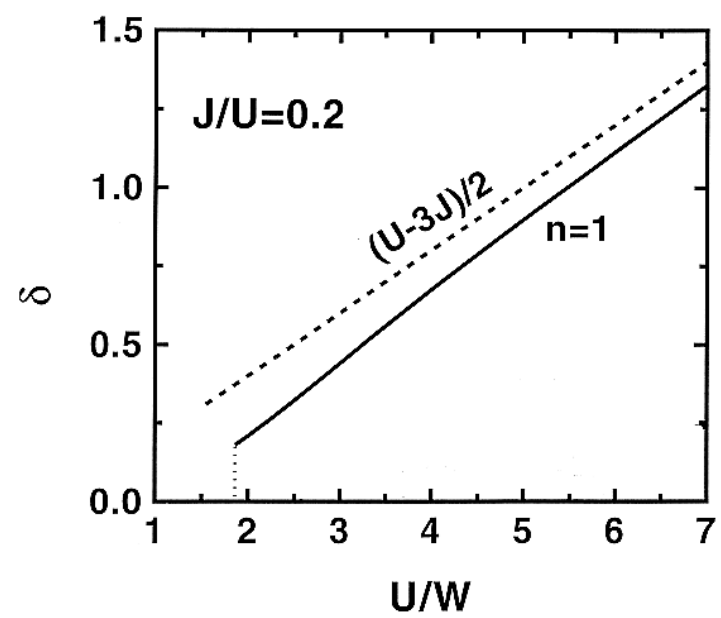

Figure 9: The half-gap parameter $\delta$ (in units of the bare bandwidth $W$ ) vs $U / W$ for a quarter-filled doubly-degenerate band. The Hund's rule exchange is $J=0.2 U$.

supported by KBN Grant No. 2 P03B 050 23. Two of the authors (ML and JS) acknowledge also the support of the Project franco-polonais "Polonium" in the period 1998-2000.

\section{References}

[1] See: J. des Cloizeaux, J. Phys. Radium. 20, 606 (1959), and D.R. Penn, Phys. Rev.142, 350 (1966), - for the stability of antiferromagnetic (AF) phases in the Hartree-Fock (HF) approximation. The stability of AF in the present context (i.e. in the Gutzwiller approximation) has been obtained in: K.Kubo and M. Uchinami, Prog. Theor. Phys. 54, 1289 (1975). For a comparative analysis see: e.g. A.M. Oleś and J. Spałek Z. Phys.B 44, 177 (1981); see also: G. Seibold et al., Phys. Rev. B 57, 6937 (1998). The phase diagram recovering both $\mathrm{HF}$ and strong correlation limits correctly has been discussed in G. Kotliar and A.E. Ruckenstein, Phys. Rev. Lett. 57, 1362 (1986)-within the slave-boson approach; and in: W. Metzner and D. Vollhardt, Phys. Rev. Lett. 62, 324 (1989); P. Fazekas, B. Menge and E. Müler-Hartmann, Z. Phys. 78, 69 (1990)-in the limit of infinite dimension $d \rightarrow \infty$ ). The corresponding discussion in two-spatial dimensions was performed in: E. Arrigoni and G.C. Strinati, Phys. Rev. B44, 7455 (1991); W. Ziegler et al., Phys. Rev. B 53, 1231 (1996).

[2] In that respect the AF stability should be considered for $\neq 1$ against the onset of the ferromagnetism, for which the Pauli principle plays the same 
role as $U \rightarrow \infty$ for antiferromagnetism. However, in the large- $U$ limit, the virtual hopping processes, particularly for $n=1$, lead to a stable AF state; see e.g. J.Spałek, A.M. Oleś, and K.A. Chao, phys. stat. solidi (b) 108, 329 (1981). Cf. also: K. A.Chao, J. Spaek, and A.M. Oleś, J. Phys. C10, L271 (1977), where the effective kinetic exchange has been derived in the $W / U \ll 1$ limit for an arbitrary $n$. Asymptotic values of the magnetic moment and the molecular-field parameter $\beta$ are discussed in: B. Möller et al., J. Phys.: Condens. Matter 5, 4847 (1993).

[3] A. Klejnberg and J. Spaek, Phys. Rev. B61, 15542 (2000); ibid., 57, 12041 (1998).

[4] Cf. also in F. Gebhardt, The Metal-Insulator Transition, Springer Tracts in Modern Physics, vol. 137 (1997).

[5] See e.g.: P. Korbel, J. Spaek, W. Wójcik, and M. Acquarone, Phys. Rev. B52, R2213 (1995).

[6] T. Li et al., Phys. Rev. B40, 6817 (1989); R. Frésard and P. Wölfle, Int. J. Mod. Phys. B6, 237 (1992); 6, 3087(E) (1992); W. Ziegler et al., in Ref[1].

[7] J. Spałek and W. Wójcik, in Spectroscopy of the Mott Insulators and Correlated Metals, Springer Series in Solid State Sciences, vol. 119, pp.41-65 (1995).

[8] M. Lavagna, Phys. Rev. B41, 142 (1990); Int. J. Mod. Phys. B5, 885 (1991).

[9] X. Yao, J.M. Honig, T. Hogan, C. Kanewurf, and J. Spaek, Phys. Rev. B54, 17469 (1996).

[10] M. Jarrell, Phys. Rev. Lett. 69, 168 (1992); M. Jarrell and T. Pruschke, Z.Phys. B90, 187 (1993). For the comparison the men-field slave-boson and the quantum Monte-Carlo approaches see: L. Lilly et al., Phys. Rev. Lett. 65, 1379 (1990).

[11] J. Spałek et. al., Phys. Rev. Lett. 48, 729 (1987); for review see: J. Spałek, J. Solid State Chem. 88, 70 (1990).

[12] For review see: A. Georges et. al., Rev. Mod. Phys. 68 , 13 (1996).

[13] The Gaussian fluctuations in PARA state are treated in Ref. 6, and in: P. Wölfle and T. Li, Z. Phys. 78, 45 (1990); R. Raimondi and C. Castellani, Phys. Rev. B48, 11453 (1993). For recent treatment see: R. Frésard and T. Kopp, Nucl. Phys. B 594, 769 (2001); A. Tandon et al., Phys. Rev. Lett. 83, 2046 (1999).

[14] R.M. Ribeiro-Teixeira and M. Avignon, in New Trends in Magnetism, Magnetic Materials, and Their Applications, edited by J. L. Moran-López and J.M. Sanchez (Plenum Press, New York, 1994) p. 373. 
[15] H. Hasegawa, J. Phys. Soc. Jpn., 66, 3522 (1997); Phys. Rev. B56, 1196 (1997); A. Klejnberg and J. Spaek, Phys. Rev. B57, 12041 (1998).

[16] A. Avella, F. Mancini and R. Münzer, Phys. Rev. B63, 245117 (2001).

[17] M. Cyrot, Phil. Mag. 25, 1031 (1972), J. Phys (Paris) 33, 125 (1972); T. Hermann and W. Nolting, J. Magn. Magn. Mat. 170, 253 (1997).

[18] These effects are considered mainly in the context of large-U situation for a two dimentional case, cf. e.g. P. Wróbel and R. Eder, Phys. Rev. B58, 15160 (1998), and references therein; cf. also: M. Imada et al., Rev. Mod. Phys. 70, 1039 (1998).

[19] P. Korbel, Ph.D. thesis (Jagiellonian University - Kraków, unpublished). 


\section{Figure Captions}

- Fig.1. The Slater gap $2 \Delta / W$ and the Hubbard gap as the functions of interaction strength. The Slater gap merges with the Mott-Hubbard gap as $U / U_{c} \rightarrow \infty$.

- Fig.2. Ground state energy for AF state and $n=1$ versus $/ U_{c}$. The lower curve is the fit to the expression $\left(-0.042 \times U_{c} / U\right)$. The inset displays the difference in behavior of magnetic moment $m$ and half of the Slater gap $(\Delta / W)$, both plotted as a function of the band filling.

- Fig.3. The Slater gap parameter $\Delta / W$ (top panel) and the magnetic moment $m=\left\langle n_{i \uparrow}-n_{i \downarrow}\right\rangle$ (bottom) versus $U / U_{c}$ and for different values of $n$.

- Fig.4. The double occupancy $d^{2}=\left\langle n_{i \uparrow} n_{i \downarrow}\right\rangle$ vs $U / U_{c}$, and for the different $n$ values. The inset display the difference in behavior for para- and antiferromagnetic cases for $n=1$.

- Fig.5. The effective mass enhancement $m^{*} / m_{0}$ (with respectto the band value $\left.m_{0}\right)$ vs $U / U_{c}$ and for the $n$ values shown. The inset shows a rather weak enhancement close to the Mott-Hubbard limit.

- Fig.6. The stability regime of AF solution; the inset: results ofMonteCarlo calculations in the $\rightarrow$ limit [8].

- Fig.7. Ground state energy vs $U / U_{c}$ for different band filling $n$. In each case the energy is $\sim U$ for $U \lesssim 0.5 U_{c}$, and $\sim 1 / U$ for $U>U_{c}$.

- Fig.8. Comparison of the magnetic $\left(-\beta_{m} m\right)$ and the Coulomb $\left(U d^{2}\right)$ terms in the ground state energy versus $U / U_{c}$.

- Fig.9. The half-gap parameter $\delta$ (in units of the bare bandwidth $W$ ) vs $U / W$ for a quarter-filled doubly-degenerate band. The Hund's rule exchange is $J=0.2 U$. 